herangezogen wird. Daraus ist zu ersehen, daß nach dem Vorschlag von Houska und Stein ${ }^{4}$ Gitterparametermessungen allein zwar durchaus brauchbare Werte für die mittleren Verschiebungsquadrate erbringen, andererseits aber Intensitäts- und Gitterparametermessungen zusammen, wie sie von DünNer und KohlHaAs ${ }^{3}$ durch- geführt wurden, umfassendere Aussagen über den Temperaturverlauf von $\overline{u_{\mathrm{s}}{ }^{2}}$ zulassen.

Für sein Interesse und die Förderung dieser Arbeit möchten wir Herrn Priv.-Doz. Dr. Rudolf Kohlhaas herzlich danken.

\section{Bruchfrontbegradigung nach Störung durch eingelagerte Teilchen und Blasen im Glas}

\section{K. Peter}

Physikalisches Laboratorium Mosbach*

(Z. Naturforsch. 23 a, 1681-1682 [1968] ; eingeg. am 5. September 1968)

Schallfraktographie ${ }^{1}$ ermöglicht Sichtbarmachung von Fronten eines spröden Bruches durch schwache periodische Ablenkungen aus der Bruchrichtung. Bruchfronten sind dabei Linien gleier Beschallungsphasen, die bis zu einem Abstand von einigen hundert $\AA$ herab aufgelöst werden konnten ${ }^{2}$. Die Experimente sind so durchführbar, daß keine wesentlichen Störungen des Bruchverlaufes durch diesen Eingriff erfolgen ${ }^{3}$.

Im einachsigen Zugversuch, dem ein homogenes Material mit seitlichem Anriß unterworfen wird, sind die Bruchfronten im genügenden Abstand vom Rand Geraden. Das stimmt um so besser, je kleiner der beobachtete Bereich im Vergleich zur Probe ist. Die Bruchfront wird durch eingelagerte Teilchen oder auch leere Zwischenräume beeinflußt ${ }^{4}$, doch stellt sich unabhängig von Größe und Art der Störung nach kurzer Laufstrecke die Geradlinigkeit der Bruchfront wieder her. Abb. $1^{* *}$ zeigt den Bruchdurchgang durch ein größeres Fluoridteilchen in Glas, die kleineren brauchen nicht beachtet zu werden. Hinter dem Teilchen ist der Bruch gegenüber dem Nachbarbereich etwas weiter vorangekommen, läuft nun aber langsamer, so daß nach wenigen $\mu$ sec (ein Linienabstand $\triangleq 1 \mu$ sec) wieder eine gerade Front entstanden ist: Der Bruch hat die Störung „vergessen“. In Abb. 2 bewirkt eine Vielzahl von Kieselglaspartikeln in Glasmatrix Abweichungen von der Art, daß hinter jedem Teilchen der Bruch gegenüber dem Nachbarbereich zurückbleibt, danach aber den Rückstand durch erhöhte Geschwindigkeit einholt. An diesem Verhalten ändert sich nichts, wenn ein bisher spontaner Bruch auf Grund der Nichterfüllung der Griffith-Bedingung ${ }^{5}$ kurzzeitig in einen thermisch bedingten Bruch übergeht. Dieser Bereich ist in Abb. 3 am Verschwinden der regelmäßigen Modulation erkennbar. Die Wirkung der hier gezeigten „Störstellen“ erstreckt sich nur wenig in Richtung der Bruchausbrei-

* Angeschlossen der Arbeitsgemeinschaft Industrieller Forschungsvereinigungen (AIF) und der Universität Karlsruhe (V 124/68).

1 F. КеккноF, Tagungsbuch über die mechanischen Eigenschaften des Glases, Symposion Florenz 1961, S. 799.

2 K. PEter, Z. Naturforsch. 20 a, 168 [1965].

3 H. Küppers, Wiss. Ber. Nr. 4/66 d. Ernst-Mach-Institutes Freiburg i. Br. 1966. tung, wohl aber weit senkrecht dazu. Die Bruchfronten haben eine gewisse Steifigkeit.

Zum Verständnis dieser Erscheinungen können Ưberlegungen zur spezifischen Bruchenergie (= „Rißausbreitungskraft") $\mathfrak{G}$ bzw. zum Spannungsverstärkungsfaktor $\Omega$ bei gekrümmter Bruchfront in einem sich unendlich erstreckenden Körper unter einachsiger Zugspannung herangezogen werden, wobei $\mathfrak{G} \sim \mathfrak{R}^{2}$ ist. Nach ${ }^{6}$ muß dann ein elliptischer Innenriß in einen Kreis übergehen, d. h. (5) und damit auch die Bruchgeschwindigkeit $^{3}$ ist um so größer, je schwächer die Bruchfrontkrümmung ist. $\mathfrak{G}$ als Funktion der Krümmung allein darzustellen, ist allerdings selbst in diesem einfachen Modell nicht möglich, doch läßt sich das Bruchverhalten nun qualitativ verstehen. Für negative Krümmungen wie in Abb. 2 ist das Modell eines ringsum angekerb-

a)

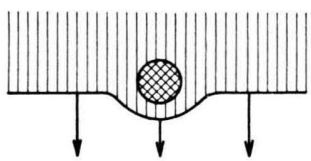

b)

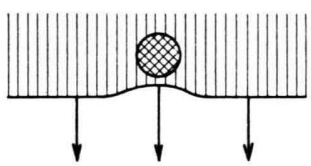

Abb. 4. Schema der Bruchfronten nach Durchgang durch eine Störung. Die Größe der Pfeile ist ein Maß für die Rißausbreitungskraft an dieser Stelle. a) entspricht Abb. 1; b) entspricht Abb. 2 (und Abb. 3).

ten Stabes brauchbar (vgl. Abb. 4). In ${ }^{7}$ ist für den reduzierten Spannungsverstärkungsfaktor

$$
\chi=\Re / \sigma \sqrt{a}
$$

( $\sigma=$ äußere Zugspannung, $a=$ Kerblänge) eine Formel zu entnehmen, die in Abb. 5 (Kurve I) aufgetragen ist. $\chi=1$ bedeutet geradlinige Bruchfront. Mit wachsendem $a$ (stärkere negative Krümmung) nimmt somit die Bruchgeschwindigkeit zu, was analog den Verhältnissen in Abb. 2 bzw. Abb. 4 b ist. Der Einfluß des lokalen zu-

${ }^{4}$ K. Peter, Z. Angew. Phys., im Druck.

* Abb. 1 bis 3 auf Tafel S. 1682 a.

5 A. A. Griffith, Phil. Trans. Roy. Soc. London A 221, 163 [1920].

${ }^{6}$ G. R. Irwin, Trans. ASME 29 E, 651 [1962].

7 P. C. Paris u. G. C. Sir, ASTM Spec. Techn. Publ. No. 381, 30 [1965]. 
sätzlichen Spannungsfeldes unmittelbar um die Störstelle herum kann dort wegen seins raschen radialen Abfalls vernachlässigt werden.

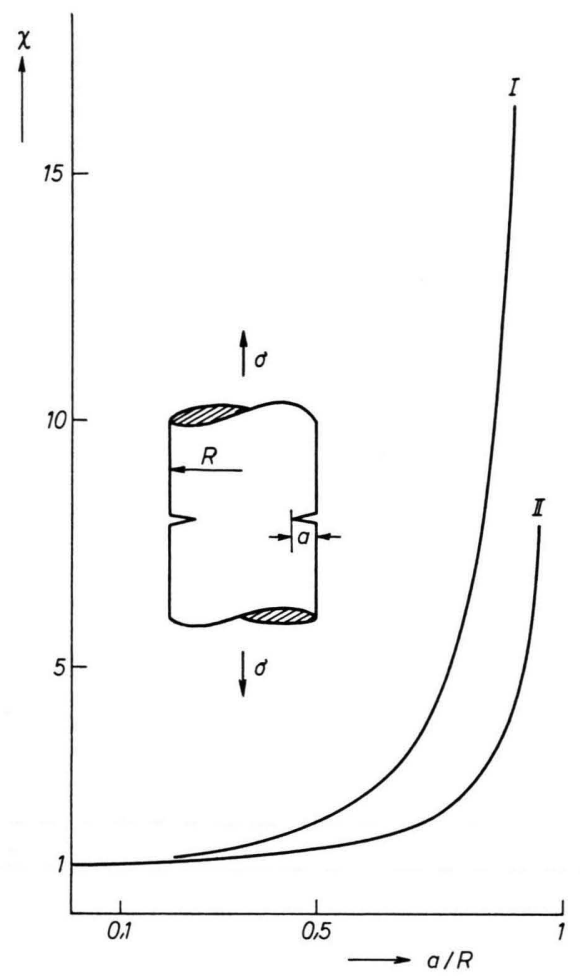

Abb. 5. Reduzierter Spannungsverstärkungsfaktor beim angekerbten Stab. Kurve I: Theoretische Abhängigkeit nach ${ }^{7}$; Kurve II: Näherung nach Formel (3).

$\mathrm{Zu}$ relativ einfachen Formeln für $\Omega$, die eine qualitative Beschreibung der Bruchfrontbegradigung ermöglichen, führt folgende Methode: Nach ${ }^{8}$ und ${ }^{9}$ ist im Fall

8 G. R. Irwin, Handb. Physik 6, 551 [1958].

9 F. C. Frank u. B. R. Lawn, Proc. Roy. Soc. London A 299, 307 [1967]. einer geradlinigen Bruchfront

$$
\mathfrak{R}=\frac{2}{\pi} \sqrt{a} \int_{0}^{a} \frac{\sigma(x) \mathrm{d} x}{\sqrt{a^{2}-x^{2}}} ;
$$

$\sigma(x)=$ Spannung am Ort $x$ auf der Kerbe vom Kerbbeginn an gerechnet, $\sigma(x) \mathrm{d} x=$ Kraft pro Längeneinheit parallel zur Bruchfront, $\sigma(x)=$ const beschreibt eine konstante äußere Zugspannung ${ }^{8}$. Bei kreisförmigem Innenriß (Radius $a$ ) betrachtet man die Beiträge $\sigma(x)$ zu einem $\Re^{*}$, die aus einem sektorförmigen „Einzugsgebiet" stammen, wobei zu $x$ ein Bogenstück $l(x)=x \mathrm{~d} \varphi$ gehört. Nach Normierung auf die Einheitslänge der Bruchfront ist

$$
\Re=\frac{2 \sqrt{a}}{\pi l(a)} \int_{0}^{a} \frac{\sigma(x) l(x) \mathrm{d} x}{\sqrt{a^{2}-x^{2}}} .
$$

Für $\sigma(x)=\sigma=$ const ergibt dies den bekannten Sneddonschen Ausdruck für den kreisförmigen Innenriß:

$$
R=(2 / \pi) \sigma \sqrt{a} \text {. }
$$

Die Methode des sektorförmigen Einzugsgebietes läßt sich auch auf den ringsum angekerbten Stab anwenden, solange die Kerbtiefe $a$ klein gegen den Stabradius $R$ ist, da die zugrunde liegende Formel (1) streng nur für sich unendlich erstreckende Körper gilt. Man erhält aus (2) mit $l(x)=(R-x) \mathrm{d} \varphi$ und $\sigma(x)=$ const

$$
\chi=\frac{1-(2 a / \pi R)}{1-(a / R)} .
$$

$R \gg a$ liefert erwartungsgemä $\Omega=\sigma \sqrt{a}$, den Wert für eine geradlinige Bruchfront. In Abb. 5 (Kurve II) ist (3) dargestellt. Näherungsweise auf beliebig gekrümmte Bruchfronten angewandt besagen diese Ausdrücke für $\chi$ bzw. $R$ : An einem Bruchfrontelement wirkt eine um so größere Rißausbreitungskraft, je geringer die Krümmung ist; dabei sind auch negative Krümmungen zu berücksichtigen. Die gesamte Bruchfront stabilisiert sich selbst zu einer Geraden.

Für Unterstützung dieser Arbeit danke ich dem Bundesministerium für Wirtschaft und der Arbeitsgemeinschaft für Elektronenoptik. 
K. PEter, Bruchfrontbegradigung nach Störung durch eingelagerte Teilchen und Blasen im Glas (S. 1681).
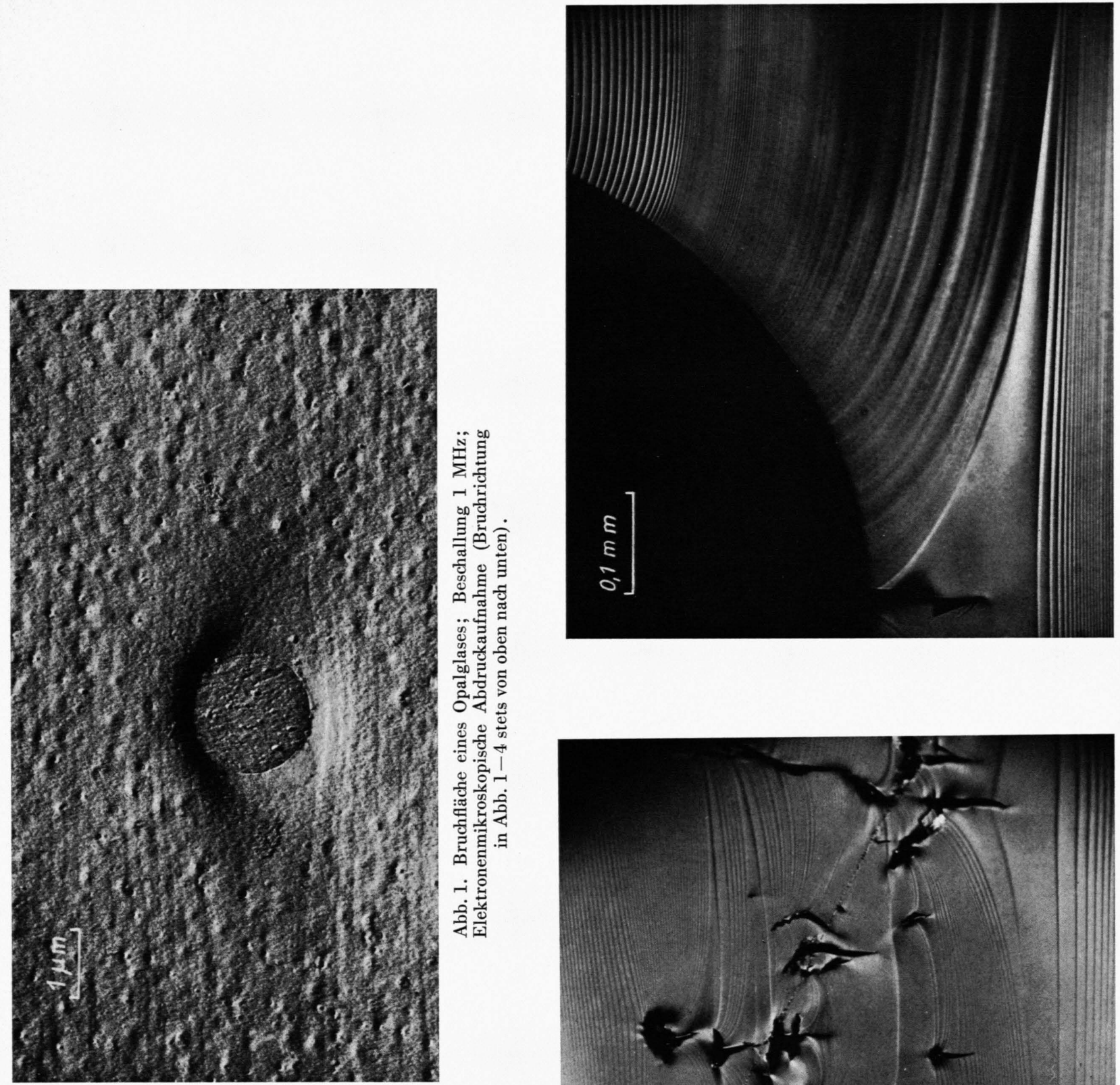

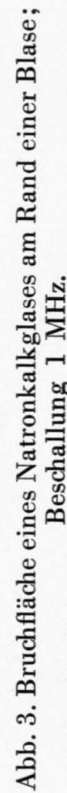
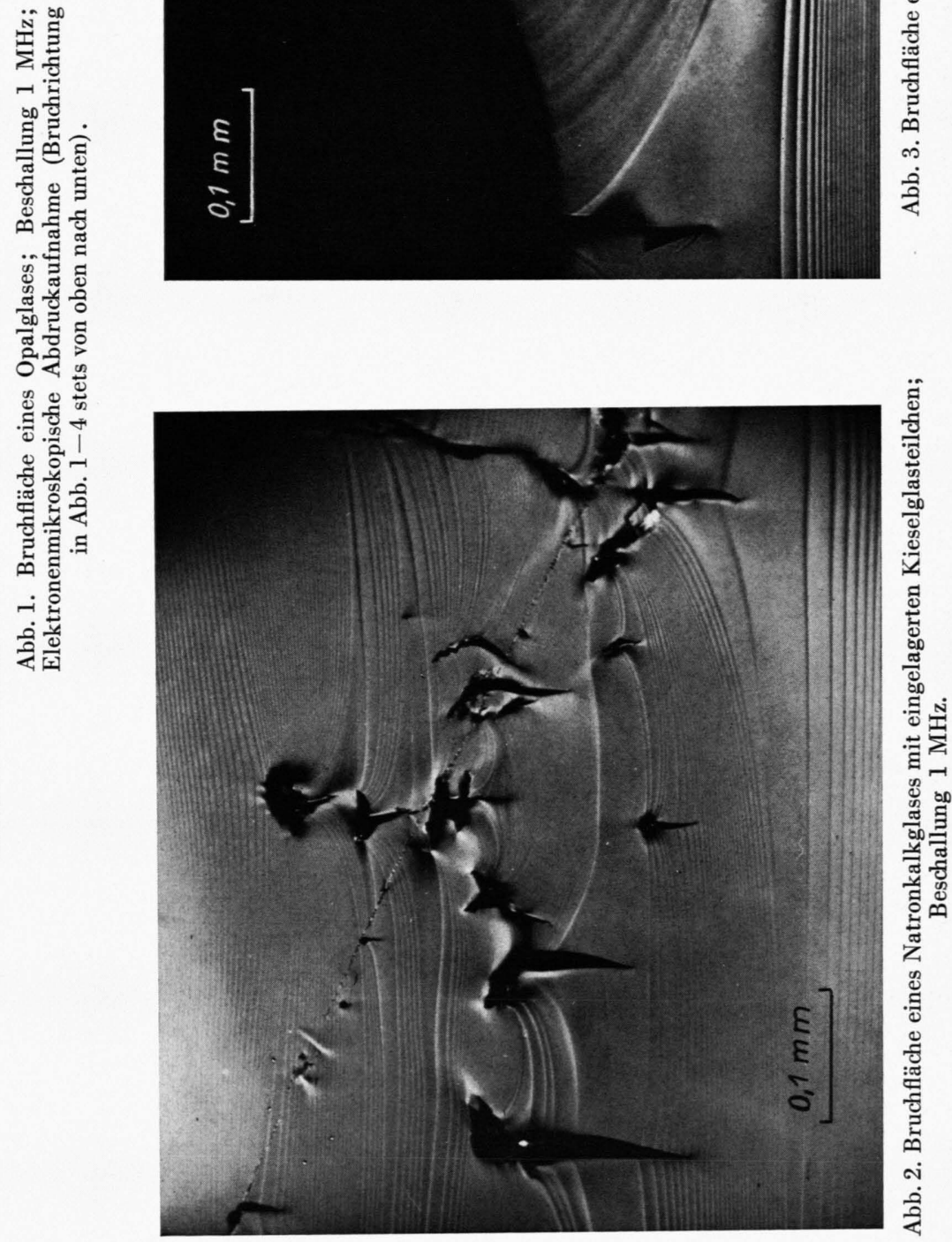
